\title{
Resiliência e vulnerabilidade em crianças que trabalham e vivem na rua
}

\author{
Sílvia Helena Koller
}

\section{RESUMO}

\begin{abstract}
O estudo dos conceitos de resiliência e vulnerabilidade vem sendo enfatizado na literatura em Psicologia, visando explicar a influência destes aspectos no desenvolvimento da pessoa. Fatores de risco e de proteção precisam ser identificados para proceder a esta avaliação. É fundamental que estes estudos sejam desenvolvidos com crianças, adolescentes e famílias em situação de rua, buscando obter dados que identifiquem os aspectos saudáveis preservados nestes grupos. É importante, ainda, subsidiar programas de fortalecimento para um desenvolvimento psicológico adaptado e de qualidade.

Palavras-chave: resiliência, vulnerabilidade, população em situação de rua.
\end{abstract}

\begin{abstract}
The study of resiliency and vulnerability concepts has been emphasized in Psychology, aiming at an answer to these two issues, which may influence someone's development. Factors of risk and protection should be identified. Such studies must be developed when one is dealing with children, teenagers and families living on the streets; healthy aspects which can lead to data should be searched. A stronger and qualified psychological development should be subsidized.

Key-words: resiliency, vulnerability, population living on the streets.
\end{abstract}

\section{Introdução}

Muitos estudos vêm demonstrando as dificuldades que as crianças em situação de rua encontram em nosso país (FORSTER et al., 1992; KOLLER ; HUTZ, 1996; MACIEL, 1997; MARTINS, 1996; VASCONCELOS, 1996, entre vários outros). Entre estas dificuldades está o alto nível de stress enfrentado por eles e o elevado número de riscos aos quais estão expostos (KOLLER et al., 1997). Stress e/ou riscos podem ser definidos como eventos de vida percebidos pela pessoa como perigosos para seu bem-estar físico e/ou psicológico (ATKINSON, et al., 1996).

Vários estudos sobre crianças em situação de risco enfatizam o efeito do stress e sua relação com a psicopatologia infantil e os antecedentes de doença mental. Recentemente, estudos que focalizam a saúde e o desenvolvimento normal têm sido desenvolvidos, mesmo com populações em situação de alto risco (HUTZ; KOLLER, 1997; RUTTER, 1985, 1987, 1993).

Tais estudos visam descrever o contexto ecológico no qual o indivíduo se desenvolve (BRONFENBRENNER, 1979,1996) e a capacidade de resiliência diante de situações que poderiam representar riscos à saúde. Resiliência tem sido definida como a capacidade dos indivíduos em emitir uma ação com um objetivo definido e como uma estratégia para alcançá-lo, diante de ou durante uma situação de risco (HOPPE; KOLLER, 1997). A pessoa resiliente mantêm-se adaptada e enfrenta com sucesso as demandas de seu desenvolvimento.

O termo resiliência foi importado das Ciências Exatas, onde é conceituado como a capacidade de um corpo físico de absorver energia, quando elasticamente deformado e, em seguida, devolver essa energia quando descarregado. Deformação elástica é definida como a capacidade de um corpo físico de retornar a sua forma original (MOURA BRANCO, 1985). Uma bola de borracha que for submetida a uma força física, volta a sua forma original quando esta força é retirada, revelando a resiliência deste material. Uma bola de argila ao 
ser submetida a uma força física, mostra as marcas do estressor, mesmo depois de retirada a força. É importante notar, no entanto, que esta importação do conceito, deve ser adaptada para a Psicologia. Uma pessoa em desenvolvimento, que sofre um estressor, não volta à forma original, porque a experiência do evento se soma as suas experiências anteriores. Este diferencial desvela-se após a retirada do estressor. Se a pessoa for frágil, ficará mais vulnerável, se for mais forte e contar com fatores de proteção, mostrará sua resiliência.

Segundo LUTHAR (1993) há três tipos de resiliência: acadêmica, social e emocional. Esta divisão é arbitrária e apenas didática e pode não abarcar todos os tipos de resiliência, uma vez que este é, ainda, um tópico recente de pesquisa e muito há que ser estudado a respeito. As áreas de resiliência mencionadas podem, no entanto, ser evidenciadas no cotidiano das pessoas. A resiliência acadêmica pode ser observada pelo bom desempenho escolar e interesse nas tarefas escolares e culturais. A resiliência social aparece no bom relacionamento interpessoal, competência social, capacidade de empatia e senso de pertencimento dos indivíduos. A resiliência emocional pode ser identificada em indivíduos com senso de auto-eficácia, autoestima e confiança em suas potencialidades, bem como no conhecimento de suas limitações (LUTHAR, 1993; ZIMMERMANN; ARUNKUMAR, 1994). Pessoas expostas a situações de risco que não desenvolvem a capacidade de resiliência são vistas como mais vulneráveis a estes eventos. Evidenciam alterações aparentes no desenvolvimento físico e/ou psicológico quando submetidas a estressores e a riscos. Tais alterações ficam evidentes na trajetória de adaptação desta pessoa, podendo torná-la suscetível e propensa a apresentar sintomas e doenças. É importante salientar que as pessoas podem ser ora vulneráveis, ora resilientes diante de um mesmo tipo de evento. Podem, ainda, ser vulneráveis em algumas áreas do seu desenvolvimento e resilientes em outros. Muitos fatores interagem no aumento da vulnerabilidade ou na redução dos efeitos do stress sobre a pessoa.

Alguns mecanismos mediadores podem influenciar na resposta da pessoa a uma situação de risco. Estes mecanismos são chamados de fatores de proteção e têm sido identificados como aqueles que reduzem o impacto de risco e de reações negativas em cadeia. As características individuais, como auto-estima e autoeficácia, são algumas delas. As oportunidades apresentadas às pessoas em situação de risco também podem influenciar na forma como elas lidam com o stress, reduzindo seu impacto e as reações negativas em cadeia. Apresentando fortalecimento (empowerment) para vencer momentos críticos da vida, aproveitando oportunidades. Os fatores de proteção mais importantes mencionados na literatura são: características de personalidade, coesão familiar e sistemas externos de apoio (MASTEN; GARMEZY, 1985). A resiliência acadêmica pode ser aumentada pelo fortalecimento de habilidades de resolução de problemas, aprendizagem de novas estratégias, capacitação de professores para auxiliar estudantes mais vulneráveis. A resiliência emocional pode ser promovida pela auto-estima e auto-eficácia, oferecimento de oportunidades de sucesso e capacitação no desenvolvimento de competência social. A resiliência social pode ser desenvolvida pela promoção de relações de amizades, participação em grupos de trabalho e esportivos e desenvolvimento do senso de pertinência ao grupo.

A avaliação de resiliência deve consistir em um processo cuidadoso através do tempo. A maioria dos resultados obtidos são produtos de estudos longitudinais, nos quais foram avaliados os eventos de risco aos quais as pessoas estavam expostas, as características de personalidade, tais como empatia, competência, locus de controle; as relações familiares, tais como vínculos, coesão, conflitos; e as redes de apoio social externo à família, como grupos religiosos, sociais, comunitários, entre outros (WERNER, 1989, WERNER; SMITH, 1992).

Alguns autores (APTEKAR, 1989; DONALD; SWART-KRUGER, 1994; HUTZ; KOLLER, 1997) sustentam que crianças em situação de rua podem ser resilientes, uma vez que apresentam caraterísticas psicológicas sadias, apesar do cotidiano adverso que enfrentam na hostilidade da rua. Elas desenvolvem estratégias para lidar com estas situações de risco. A rua gera altos níveis de stress, testando constantemente sua vulnerabilidade emocional, social e cognitiva. Todavia, também, exige permanentemente que elas apresentem estratégias de adaptação e de resistência à situação adversa e que põe em risco a sua segurança e sobrevivência.

\section{REFERÊNCIAS}

APTEKAR, L. Colombian street children Gamines or Chupagruesos. Adolescence, n. 24, p. 783-794.

ATKINSON, R. L.; ATKINSON, R. C. et al. Introdução à psicologia. Porto Alegre: Artes Médicas, 1996. 
BRONFENBRENNER, U. A ecologia do desenvolvimento humano: experimentos naturais e planejados. Porto Alegre: Artes Médicas, 1996 (Original publicado em 1979).

DONALD, D.; SWART-KRUGER, J. The South-African street child: developmental implications. South-African Journal of Psychology, n. 24, p. 169-174, 1994.

FORSTER, L. M. K.; BARROS, H. M. T. et al. Meninos de rua: relação entre abuso de drogas e atividades ilícitas. Revista da ABP-APAL, n. 14, p. 115-120, 1992.

HOPPE, M.; KOLLER, S. H. Redes de apoio social e afetivo de crianças de nível socioeconômico baixo. CONGRESSO INTERAMERICANO DE PSICOLOGIA (26.:1997: São Paulo). Anais... São Paulo, 1997.

HUTZ, C.; KOLLER, S. Questões sobre o desenvolvimento de crianças em situação de rua. Estudos de Psicologia, n. 2 , p. $175-197,1997$.

HUTZ, C.; KOLLER, S. H.; BANDEIRA, D. R. Resiliência e vulnerabilidade em crianças em situação de risco. Coletâneas da ANPEPP, v. 1, n. 12, p. 79-86, 1996.

KOLLER, S. H.; HUTZ, C. Meninos e meninas em situação de rua: dinâmica, diversidade e definição. Coletâneas da $A N P E P P$, v. 1, n. 12, p. 11-34, 1996.

KOLLER, S. H., RAFFAELLI, M. et al. Using a sentence completion task to investigate how Brazilian street youth describe their life situation. 62nd. Washington, DC: Society for Research in Child Development, 1997.

LUTHAR, S. S. Annotation: methodological and conceptual issues in research on childhood resilience. Journal of Child Psychology and Psychiatric, v. 34, p. 441-453, 1993.

MACIEL, C. Explicações das desigualdades sociais dadas por meninos de rua em João Pessoa. João Pessoa, 1997. Dissertação (Mestrado em Psicologia) - Universidade Federal da Paraíba. No prelo.

MARTINS, R. A. Crianças e adolescentes em situação de rua: definições, evolução e políticas de atendimento. Coletâneas da ANPEPP, v. 1, n. 12, p. 35-44, 1996.

MASTEN, A. S.; GARMEZY, N. Risk, vulnerability, and proctective factors in developmental psychopathology. In: LAHEY, B. B.; KAZDIN, A. E. (Orgs.). Advances in Clinical Child Psychology. New York: Plenum Press, 1985. v. 8.

MOURA BRANCO, C. A. G. Mecânica dos materiais. Lisboa: Fundação Calouste Gulbenkian, 1985.

RUTTER, M. Resilience in the face of adversity: protective factors and resistance to psychiatric disorder. British Journal of Psychiatric, n. 147, p. 598-611, 1985.

RUTTER, M. Psychosocial resilience and protective mechanisms. American Journal of Orthopsychiatry, n. 57, p. $316-$ $331,1987$.

RUTTER, M. Resilience: some conceptual considerations. Journal of Adolescent Health, n. 14, p. 626-631, 1993.

VASCONCELOS, M. M. P. Família, trabalho e drogas: estudo exploratório da representação dos meninos de rua da cidade de João Pessoa sobre as suas condições de vida. João Pessoa, 1996. Dissertação (Mestrado em Psicologia) Universidade Federal da Paraíba. No prelo.

ZIMMERMAN, M.; ARUNKUMAR, R. Resiliency research: implications for schools and policy. Social Policy Report, n. 8, p. 1-18, 1994.

WERNER, E. E. High-risk children in young adulthood: a longitudinal study from birth to 32 years. American Journal of Orthopsychiatry, n. 59, p. 72-81, 1989.

WERNER, E.; SMITH, R. Overcoming the odds. New York: Cornell University Press, 1992. 